\section{P81 Extension Nutrition Educators and Community Stakeholders Perceive the Healthfulness of Their Under-Resourced Florida Communities Differently}

Jamie Zeldman, MS, RDN, University of Florida; Sydnee Berman, University of Florida; Elder Varela, MA, MS, CHES, University of Florida; Karla Shelnutt, PhD, RD, kpagan@ufl. edu, University of Florida, 3028 McCarty Hall D, Gainesville, FL, 32611

Background: Community stakeholders and Extension nutrition educators (NEs) are invested in the health of their under-resourced communities and should be included in the development of interventions designed to improve the health of their residents. These groups understand barriers and facilitators to healthy living that can help inform appropriate health interventions. As perceptions can differ, input from both groups can provide a more well-rounded approach.

Objective: To compare Extension NE and community stakeholder perceptions on the healthfulness of the environment of under-resourced communities in the 5 Florida Extension districts

Study Design, Settings, Participants: Focus groups (n $=8)$ with Extension NEs $(\mathrm{N}=49)$ and in-depth interviews $(\mathrm{n}=10)$ with community stakeholders were conducted using semi-structured scripts following the Social Cognitive Theory to explore perceptions on the healthfulness of the communities.

Measurable Outcome/Analysis: Focus groups and indepth interviews were audio-recorded, transcribed verbatim, and coded. Cross-tab analysis was used to compare data from the districts' participants.

Results: Overall, participants described healthy eating and physical activity as the main components of a healthy lifestyle. They also viewed access to and availability of food as an essential part of a healthy community. While both NEs and stakeholders perceived food access (Northwest, Southwest), safety (Central), and lack of education (Southeast) as major barriers to a healthy lifestyle, NEs also identified lack of knowledge and education as major barriers, whereas stakeholders perceived financial constraints (ie, unemployment) as the primary challenge. When asked about resources available to address challenges, NEs (all districts) did not perceive many, which they attributed to a lack of promotion of resources. In contrast, stakeholders (all districts) perceived multiple opportunities available but noted a lack of participation from community members.

Conclusion: Extension NEs and community stakeholders provide unique perspectives about barriers and opportunities in the under-resourced communities they serve. Differences among the districts and between participants highlight diverse characteristics of these communities, which should be considered when developing interventions.

Funding: UF IFAS Office of Research.

\section{P82 Food Neophobia Among Korean Young Adult in Their 20s Focusing on its Association with Fruit and Vegetable Preference, Acceptance and Perceived Intake}

So-Young Kim, PhD, sonyah@sch.ac.kr, Soonchunhyang University, 22 Soonchunhyang-ro, Asan, Chungnam, S. Korea, 31538; Meeyoung Kim, PhD, Kongju National University

Background: Food neophobia is indicated as 1 of the most critical factors affecting reduced preferences for, and lower intakes of fruits and vegetables.

Objective: This study aimed to explore whether food neophobia contributes to preference, acceptance and perceived daily intake of fruits and vegetables among Korean young adults in their 20s.

Study Design, Setting, Participants: An on-line survey was conducted from February 18-24, 2020 to Korean young adults (male: 570 , female: 707 ) in their 20s. A survey questionnaire was developed to include the food neophobia scale and to assess respondents' preference, acceptance, and perceived daily intake of fruits and vegetables in diversity and amount. Other dietary habits related to taste preferences and perceived healthiness were also measured.

Measurable Outcome/Analysis: Respondents were divided into 3 groups according to food neophobia score distribution (low, middle or high). The distributive differences among the 3 groups were analyzed using a chi-squared test according to respondents' general characteristics (gender, BMI, education level. and income level). Significant differences in vegetable and fruit consumption (ie, preference, acceptance, and perceived daily intake) were measured across the food neophobia groups by using ANOVA. All statistical analyses were performed using the SPSS program at the significance level of 0.05 .

Results: The results showed that females were more likely to be food neophobic than males. Higher level of food neophobia was significantly associated with lower preference, acceptance, and perceived daily intake of fruits and vegetables both in diversity and amount. Young adults with higher level of food neophobia were more likely to prefer salty foods, but less likely to perceive themselves to be healthy.

Conclusion: The results suggest that food neophobia may negatively influence fruit and vegetable consumption among Korean young adults.

Funding: National Research Foundation of Korea (NRF) grant funded by the Korea government (MSIT) (No. NRF2019R1G1A1100488). 